\title{
Percepção dos usuários, profiissionais de saúde e gestores sobre o modelo de atendimento oftalmológico no Hospital Regional de Divinolândia - São Paulo
}

\author{
Perception of the users, health professionals and managers of the assistance \\ model of the Regional Ophthalmologic Hospital of Divinolândia - SP
}

\author{
Maria Cecília Machado ${ }^{1}$ \\ Flávio Mac Cord Medina² \\ Newton Kara-José ${ }^{3}$
}

\begin{tabular}{|l|}
\hline RESUMO \\
\hline Objetivo: Avaliar a qualidade do atendimento, pela percepção dos usu- \\
ários e gestores municipais de saúde (prefeitos, secretários de saúde e \\
triadores). Métodos: Realizou-se um estudo transversal e descritivo. \\
Resultados: A amostra foi composta por 359 usuários e 48 gestores. O \\
atendimento médico foi considerado ótimo por $79,6 \%$ dos usuários, por \\
$93,7 \%$ dos prefeitos, por $87,5 \%$ dos secretários de saúde e por $100 \%$ dos \\
triadores. O pessoal de enfermagem foi considerado ótimo por $75,7 \%$ \\
dos usuários, 93,8\% dos prefeitos, $75,0 \%$ dos secretários de saúde e por \\
$100 \%$ dos triadores. A recepção recebeu avaliação ótima de $73,8 \%$ dos \\
usuários e 93,8\% dos triadores. Conclusão: O modelo de atendimento \\
da Clínica Oftalmológica de Divinolândia obteve alta satisfação tanto \\
dos usuários como dos gestores.
\end{tabular}

Descritores: Instituições de saúde; Administração em saúde; Serviços de saúde comunitária; Controle de qualidade; Satisfação dos consumidores

\section{INTRODUCÃO}

Pesquisa realizada na Clínica Oftalmológica de Divinolândia e na Universidade Estadual de Campinas - UNICAMP - Campinas (SP) - Brasil.

Pós-Graduanda (Mestrado) da Faculdade de Ciências Médicas da Universidade Estadual de Campinas - UNICAMP - Campinas (SP) - Brasil; Enfermeira da Clínica Oftalmológica de Divinolândia - Divinolândia (SP) - Brasil.

${ }^{2}$ Pós-Graduando (Mestrado) da Faculdade de Ciências Médicas da UNICAMP - Campinas (SP) - Brasil.

${ }^{3}$ Professor Titular de Oftalmologia da Faculdade de Ciências Médicas da UNICAMP - Campinas (SP) - Brasil.

Endereço para correspondência: Maria Cecília Machado. Rua Leonor Mendes de Barros, 501 - Divinolândia (SP) CEP 13780-000

E-mail: cecilymachado@yahoo.com.br

Recebido para publicação em 22.03.2007

Última versão recebida em 21.05.2010

Aprovação em 03.06.2010

Esta pesquisa é parte da Tese de Mestrado da autora Maria Cecília Machado na Faculdade de Ciências Médicas na Universidade Estadual de Campinas - UNICAMP - Campinas (SP) - Brasil.

Este projeto foi parcialmente financiado pelo Consórcio de Desenvolvimento da Região de Governo de São João da Boa Vista - CONDERG. 


\section{O serviço de oftalmologia de Divinolândia}

Na cidade de Divinolândia, funciona um modelo de serviço oftalmológico gerido pelo Consórcio de Desenvolvimento da Região de Governo de São João da Boa Vista (Conderg) e pela Disciplina de Oftalmologia da Universidade Estadual de Campinas (Unicamp).

Esse consórcio foi criado em 13 de julho de 1987, através de uma parceria firmada entre 16 municípios da região de São João da Boa Vista, e nesse mesmo ano, celebrou um convênio com o serviço de oftalmologia da Universidade de Campinas (Unicamp), representado pela Disciplina de Oftalmologia e pelo Prof. Dr. Newton Kara-José, com o Departamento Regional de Saúde (DRS) ${ }^{(4)}$.

O serviço tem como finalidades complementar o atendimento oftalmológico da região, aprimorar o ensino dos residentes da Unicamp e demonstrar a exequibilidade de extensão dos serviços de hospital-escola. O programa é financiado pelo SUS sendo que o atendimento e a disponibilidade de óculos são inteiramente gratuitos. De 1987 até 2009, foram realizadas 230.970 consultas, 20.413 cirurgias e doados 55.313 óculos

A Clínica Oftalmológica de Divinolândia exerce atendimento fundamentalmente de nível secundário e terciário. Cada município do consórcio tem um número definido de consultas por mês e os pacientes são triados e agendados nos centros de saúde locais, por triadores. As prefeituras providenciam o transporte gratuito até Divinolândia ${ }^{(5)}$.

A clínica vem aumentando sua capacidade de atendimento para suprir uma demanda crescente. Inicialmente, o serviço contava com recursos básicos e vem se expandindo progressivamente, com o aumento dos recursos humanos e materiais financiados pelo SUS e pelo Conderg.

\section{Óptica do Conderg}

Vícios de refração não corrigidos são a maior causa de diminuição da visão nos países em desenvolvimento, calculando-se 150 milhões em todo o mundo ${ }^{(6)}$. Apesar da fácil correção e baixo custo, o problema parece estar em constante aumento.

As barreiras para a correção óptica são multifatoriais, sendo as principais, a dificuldade de acesso ao exame oftalmológico e a aquisição de óculos. Nos EUA estima-se que 11 milhões de indivíduos necessitam e não têm correção óptica ${ }^{(7-8)}$.

No Brasil este número deve ser muito maior, pois cerca de $80 \%$ da população depende unicamente do SUS, o qual, apesar de muitos progressos, ainda convive com uma crônica falta de recursos ${ }^{(9)}$.

Relatos mostram que cerca de $30 \%$ dos pacientes da Unicamp não aviaram as receitas por falta de recursos financei$\operatorname{ros}^{(8)}$.

A óptica do Conderg proporciona o livre acesso à correção óptica dos usuários da Clínica e nesses 22 anos foram distribuídos 55.313 óculos $^{(10-11)}$.

Este trabalho tem como objetivo identificar percepções de usuários, gestores municipais de saúde e profissionais de saúde em relação ao serviço Oftalmológico do Hospital de Divinolândia, visando obter informações para avaliar e melhorar a qualidade do atendimento.

\section{MÉTODOS}

Foi realizado um estudo transversal, descritivo, na Clínica de Oftalmologia do Hospital Regional de Divinolândia, envolvendo usuários e gestores dos 16 municípios.

A pesquisa compôs-se de duas amostras, sendo a primeira não probabilística, prontamente acessível, envolvendo todos os 359 usuários, que procuraram o serviço durante uma semana considerada típica, isto é, sem feriado, greves ou ponto facultativo, que impedissem o fluxo habitual de procura $^{(12)}$.

Para os usuários menores de idade e para os idosos, foi solicitado aos acompanhantes o auxílio no preenchimento do questionário.

A segunda amostra foi composta pelos 48 gestores (prefeitos, secretários e profissionais de saúde) dos 16 municípios do Conderg.

Foram elaboradas duas versões do instrumento de pesquisa. Para os usuários foi aplicado um questionário misto, com questões fechadas e abertas, e autoaplicável. O questionário autoaplicável aplicado aos gestores foi elaborado com questões fechadas.

\section{Coleta de dados}

A pesquisa desenvolveu-se em duas fases: na primeira, foram apuradas as opiniões dos usuários e na segunda fase foram coletadas opiniões dos gestores. Os dados da primeira fase foram coletados durante a semana típica de 7 a 11 de agosto de 2006.

Os pacientes foram abordados na sala de espera da clínica sendo salientada a finalidade do estudo, sua disponibilidade (espontânea) de cooperação e concordância com a assinatura no "Termo de Consentimento".

Após responderem o questionário, os pacientes depositavam os mesmos, em uma urna, localizada ao lado da recepção. Os dados referentes aos gestores foram coletados na semana de 14 a 21 de agosto de 2006. Os questionários dos gestores e os termos de consentimento eram entregues em seus locais de trabalho.

\section{Processamento e análise dos dados}

As informações obtidas foram digitadas em um banco de dados utilizando o programa Windows (Vista). Foi realizada dupla digitação para minimizar erros.

A média aritmética simples das questões de cada bloco apurou o índice daquela dimensão. Os resultados foram apresentados em forma de tabelas e gráficos (Windows-Excel).

\section{Comitê de ética}

Esta pesquisa foi aprovada pela Comissão de Ética da Unicamp - Projeto de Pesquisa n⿳ำ 520/2006 e pela Comissão de Ética do Conderg - Projeto de Pesquisa n 670/2006. 
278 Percepção dos usuários, profissionais de saúde e gestores sobre o modelo de atendimento oftalmológico no Hospital Regional de Divinolândia - São Paulo

\section{RESULTADOS}

Quanto a se receberam orientação sobre o procedimento médico, 99,7\% usuários responderam "sim" e $0,2 \%$ responderam "não". E sobre se recomendariam este hospital para amigos, 93,9\% responderam "sim" e 6,1\% não responderam.

Forneceram sugestões sobre o espaço físico da sala de espera $20(5,5 \%)$ usuários. Treze $(3,6 \%)$ sugeriram aumento do número de médicos, $6(1,6 \%)$ gostariam que o tempo de espera para atendimento fosse menor, $5(1,3 \%)$ que fossem doados os colírios, $2(0,5 \%)$ que houvesse mais banheiros e $199(55,4 \%)$ usuários disseram não possuírem sugestões por estarem satisfeitos. Não emitiram sugestões 117 usuários (32,5\%).

Na opinião dos prefeitos, $15(93,7 \%)$ consideraram ótimos os atendimentos de médicos, enfermagem e recepção, e 1 (6,3\%) considerou bom (Tabela 2$)$.

Dos prefeitos, 7 (43,7\%) sugeriram a ampliação da capacidade de atendimento, 2 (12,5\%) gostariam que consultas e retornos fossem agendados para o mesmo dia e $1(6,25 \%)$ gostaria que o espaço físico fosse ampliado.

$\mathrm{Na}$ opinião dos secretários municipais de saúde (Tabela 3), $14(87,5 \%)$ consideraram ótimos os atendimentos de médicos e recepção, e $2(12,5 \%)$ consideraram bom. Com relação à enfermagem, $12(75 \%)$ consideraram o atendimento ótimo e 4 (25\%) bom.

Na opinião dos triadores, $16(100 \%)$ consideraram ótimo o atendimento médico e de enfermagem e $15(93,7 \%)$ a recepção (Tabela 4).

Todos os prefeitos e secretários de saúde consideraram o atendimento como ótimo e/ou bom.

\section{DISCUSSÃO}

A verificação da percepção de usuários e gestores municipais de saúde em relação ao modelo da Clínica Oftalmológica de Divinolândia, inserida dentro de um consórcio intermunicipal de saúde em parceria com a Unicamp, sempre foi realizada de maneira empírica, não possibilitando uma análise científica dos resultados obtidos. Este estudo foi motivado pela necessidade da obtenção de informações científicas para avaliar o impacto dos resultados e servir de subsídios para aprimoramento do sistema.

Uma das metas da Clínica de Oftalmologia, iniciada em 1988, foi complementar o atendimento oftalmológico da região do Conderg, visando alcançar uma cobertura total para a população ${ }^{(13)}$.

Nesse sentido, desde o início, a demanda e a capacidade de atendimento vem aumentado exponencialmente, porém os prefeitos querem uma cobertura oftalmológica ainda maior. Os obstáculos existentes para tal tarefa residem no espaço físico atual e limitação de financiamento do SUS e das prefeituras.

No momento encontra-se em fase final de construção um hospital de olhos com uma área física de $1.100 \mathrm{~m}^{2}$, o que dará condições para a ampliação do atendimento. Os resultados obtidos nessa pesquisa servirão de subsídios para a reivindicação de maior financiamento.

Um fator importante para a manutenção e aprimoramento desse projeto é o excelente relacionamento e concordância de propósitos entre a equipe de saúde e a direção do Conderg. A clínica oftalmológica já conviveu com 5 mandatos de dirigentes das 16 prefeituras da região, o que sugere isenção política alcançada no gerenciamento do serviço. A satisfação dos gestores e dos usuários tem sido de grande valia para manutenção e aprimoramento da instituição (Tabelas 1-4).

Durante esses 22 anos de funcionamento, todos os aspectos relevantes do serviço, desde reclamações até observações dos gestores e administradores do hospital, foram avaliados em reuniões e muitas mudanças foram sugeridas e realizadas. Entre as melhorias estão a criação e ampliação de uma óptica que fornece atualmente 400 unidades por mês e já distribuiu, gratuitamente, 50.520 óculos $^{(10-11)}$.

Com a aquisição de novos equipamentos, a clínica aumentou sua capacidade de resolubilidade para $96,46 \%$. A clínica de oftalmologia em 2009 atendeu 28.996 e realizou 1.779 cirurgias. ${ }^{*}$

Evidenciou-se que, com pequenas diferenças, as opiniões dos gestores (prefeitos e secretários de saúde e triadores) convergem (Tabelas 2-4). Os triadores deram uma avaliação melhor, o que deve ter ocorrido pelo contato direto que elas têm com os pacientes, os quais estão satisfeitos em sua maioria, pois todos os encaminhamentos são atendidos e, quando necessário, são adicionados casos extras (Tabela 4). Outro ponto para a boa avaliação dos pacientes, certamente se deve à continuidade do atendimento nesses 22 anos.

Pacientes tiveram a perspectiva inferior quando comparada com a dos gestores e triadores, porém com avaliação ótima em 79,6\% dos casos e bom em 19,7\% (Tabelas 1-4). Uma das queixas dos usuários é o tempo que ficam na clínica, pois a maioria chega pela manhã, utilizando transporte coletivo fornecido pelo município de origem e o retorno ocorre quando o último paciente é atendido. O ideal seria um escalonamento de chegada, porém isto traria maiores despesas e transtornos para as prefeituras municipais.

Alguns pesquisadores no Brasil, têm buscado compreender o fenômeno da alta satisfação dos usuários de serviços públicos de saúde. Enquanto uns apontam inconsistências teóricas no conceito de satisfação ${ }^{(15)}$, outros o relacionam a aspectos metodológicos ${ }^{(16-17)}$. No caso dos serviços públicos, argumenta-se, ainda, que a alta satisfação possa expressar o receio dos usuários em perder o direito ao serviço ${ }^{(18)}$. Outros fatores têm sido apontados como responsáveis pelo fenômeno da alta satisfação, tais como, a relutância em expressar opiniões negativas, conhecido como o viés de gratidão(19-20).

Esses fatores de viés estão minimizados ou anulados nessa pesquisa, pois os pacientes, após a consulta, voltam para o

* Informação do Conderg. 


\begin{tabular}{|c|c|c|c|c|c|c|c|c|c|c|c|c|}
\hline & \multicolumn{2}{|c|}{ Ótimo } & \multicolumn{2}{|c|}{ Bom } & \multicolumn{2}{|c|}{ Ruim } & \multicolumn{2}{|c|}{ Péssimo } & \multicolumn{2}{|c|}{ Não respondeu } & \multicolumn{2}{|c|}{ Total } \\
\hline & $f$ & $\%$ & $f$ & $\%$ & $f$ & $\%$ & $f$ & $\%$ & $f$ & $\%$ & $f$ & $\%$ \\
\hline Médico & 286 & 79,7 & 71 & 19,8 & 1 & 0,3 & 0 & 0,0 & 1 & 0,3 & 359 & 100,0 \\
\hline Enfermagem & 272 & 75,8 & 76 & 21,2 & 0 & 0,0 & 0 & 0,0 & 11 & 3,1 & 359 & 100,0 \\
\hline Recepção & 265 & 73,8 & 79 & 22,0 & 0 & 0,0 & 0 & 0,0 & 15 & 4,2 & 359 & 100,0 \\
\hline Total & 823 & 76,4 & 226 & 21,0 & 1 & 0,1 & 0 & 0,0 & 27 & 2,5 & 1077 & 100,0 \\
\hline
\end{tabular}

\begin{tabular}{|c|c|c|c|c|c|c|c|c|c|c|c|c|}
\hline & \multicolumn{2}{|c|}{ Ótimo } & \multicolumn{2}{|c|}{ Bom } & \multicolumn{2}{|c|}{ Ruim } & \multicolumn{2}{|c|}{ Péssimo } & \multicolumn{2}{|c|}{ Não respondeu } & \multicolumn{2}{|c|}{ Total } \\
\hline & $f$ & $\%$ & $f$ & $\%$ & $f$ & $\%$ & $\bar{f}$ & $\%$ & $f$ & $\%$ & $f$ & $\%$ \\
\hline Médico & 15 & 93,8 & 1 & 6,3 & 0 & 0,0 & 0 & 0,0 & 0 & 0,0 & 16 & 100,0 \\
\hline Enfermagem & 15 & 93,8 & 1 & 6,3 & 0 & 0,0 & 0 & 0,0 & 0 & 0,0 & 16 & 100,0 \\
\hline Recepção & 15 & 93,8 & 1 & 6,3 & 0 & 0,0 & 0 & 0,0 & 0 & 0,0 & 16 & 100,0 \\
\hline Total & 45 & 93,8 & 3 & 6,3 & 0 & 0,0 & 0 & 0,0 & 0 & 0,0 & 48 & 100,0 \\
\hline
\end{tabular}

\begin{tabular}{|c|c|c|c|c|c|c|c|c|c|c|c|c|}
\hline & \multicolumn{2}{|c|}{ Ótimo } & \multicolumn{2}{|c|}{ Bom } & \multicolumn{2}{|c|}{ Ruim } & \multicolumn{2}{|c|}{ Péssimo } & \multicolumn{2}{|c|}{ Não respondeu } & \multicolumn{2}{|c|}{ Total } \\
\hline & $f$ & $\%$ & $f$ & $\%$ & $f$ & $\%$ & $\bar{f}$ & $\%$ & $f$ & $\%$ & f & $\%$ \\
\hline Médico & 14 & 87,5 & 2 & 12,5 & 0 & 0,0 & 0 & 0,0 & 0 & 0,0 & 16 & 100,0 \\
\hline Enfermagem & 12 & 75,0 & 4 & 25,0 & 0 & 0,0 & 0 & 0,0 & 0 & 0,0 & 16 & 100,0 \\
\hline Recepção & 14 & 87,5 & 2 & 12,5 & 0 & 0,0 & 0 & 0,0 & 0 & 0,0 & 16 & 100,0 \\
\hline Total & 40 & 83,3 & 8 & 16,7 & 0 & 0,0 & 0 & 0,0 & 0 & 0,0 & 48 & 100,0 \\
\hline
\end{tabular}

\begin{tabular}{|c|c|c|c|c|c|c|c|c|c|c|c|c|}
\hline & \multicolumn{2}{|c|}{ Ótimo } & \multicolumn{2}{|c|}{ Bom } & \multicolumn{2}{|c|}{ Ruim } & \multicolumn{2}{|c|}{ Péssimo } & \multicolumn{2}{|c|}{ Não respondeu } & \multicolumn{2}{|c|}{ Total } \\
\hline & $f$ & $\%$ & $f$ & $\%$ & $f$ & $\%$ & $\bar{f}$ & $\%$ & $f$ & $\%$ & $f$ & $\%$ \\
\hline Médico & 16 & 100,0 & 0 & 0,0 & 0 & 0,0 & 0 & 0,0 & 0 & 0,0 & 16 & 100,0 \\
\hline Enfermagem & 16 & 100,0 & 0 & 0,0 & 0 & 0,0 & 0 & 0,0 & 0 & 0,0 & 16 & 100,0 \\
\hline Recepção & 15 & 93,8 & 1 & 6,3 & 0 & 0,0 & 0 & 0,0 & 0 & 0,0 & 16 & 100,0 \\
\hline Total & 47 & 97,9 & 1 & 2,1 & 0 & 0,0 & 0 & 0,0 & 0 & 0,0 & 48 & 100,0 \\
\hline
\end{tabular}

centro de saúde onde foram triados e têm livre acesso aos triadores e mesmo às autoridades municipais. Não existe o perigo de perderem o direito ao atendimento, pois a seleção é feita por pessoas diretamente ligadas à comunidade e sem dependência com os funcionários da clínica.

A equidade do atendimento da clínica é garantida pela seleção impessoal dos pacientes encaminhados pelos Centros de Saúde, não havendo, até hoje, casos de rejeição de pacientes por qualquer motivo alheio às condições de saúde ocular. A aceitabilidade do serviço pode ser aferida também pela crescente demanda. A acessibilidade é ainda facilitada pela marcação de consultas no próprio município e a oferta de transporte gratuito até Divinolândia ${ }^{(5)}$.
O atendimento médico foi avaliado como bom ou ótimo pela quase totalidade dos usuários (Tabela 1). Da mesma forma, o atendimento de enfermagem e o atendimento da recepção apresentaram alto grau de aceitação pelo serviço prestado (Tabelas 1-4). A percepção dos gestores municipais também se manifestou através dos conceitos bons e ótimos em relação ao atendimento médico, de enfermagem e de recepção (Tabelas 2-4).

Elias et al. ${ }^{(21)}$ avaliando centros e unidades básicas de saúde (UBS), detectaram que a acessibilidade a elas foi percebida por usuários e gestores de forma negativa, sugerindo dificuldades estruturais na oferta de serviços. Foram citados os rígidos e limitados horários de funcionamento e as formas quase sempre presenciais de agendamento. No modelo do 
280 Percepção dos usuários, profissionais de saúde e gestores sobre o modelo de atendimento oftalmológico no Hospital Regional de Divinolândia - São Paulo

Hospital, apesar dos horários serem determinados, através da cooperação dos triadores municipais são aceitas inclusões de casos excepcionais.

A percepção de gestores em relação ao atendimento de uma unidade de saúde (Tabelas 2-4) reflete a percepção dos usuários do sistema. A opinião de gestores de saúde é influenciada pela informação contínua provida pelos usuários, o que dá credibilidade ao dados obtidos, a partir de manifestações constantes com gestores e demais munícipes ${ }^{(21)}$.

No caso da Clínica Oftalmológica de Divinolândia, o aumento da procura, a crescente ampliação da cobertura do atendimento e a taxa de resolubilidade, certamente contribuíram para o alto grau de satisfação com o serviço prestado.

A clínica se reestrutura continuamente, estabelecendo metas anuais, que são resolvidas em reuniões mensais pela direção e replanejadas anualmente. Os resultados obtidos nesta pesquisa mostram, na opinião dos usuários e gestores, os excelentes padrões obtidos pelo serviço (Tabelas 1-4).

Este estudo evidenciou o sucesso de um modelo de associação entre universidade e um consórcio intermunicipal, podendo servir de modelo para outras universidades. A extensão de serviços à comunidade, amplia a área de cobertura de regiões carentes, ajudando na resolução de seus problemas médicos.

No estágio de Divinolândia, os residentes recebem casos novos com menor tempo de evolução, podendo exercer melhor, a prática de diagnóstico e reavaliação de condutas e acompanhamento da evolução do caso, o que geralmente não ocorre em um hospital de nível terciário. Por exemplo, Gullo et al. ${ }^{(22)}$ mostraram que a grande maioria dos pacientes que chegam no $\mathrm{HC}$ da Unicamp com diagnóstico de glaucoma, encontram-se em estágio avançado da doença.

A facilitação do atendimento no hospital de Divinolândia, com ampla cobertura das necessidades da população, acesso universalizado, alta resolubilidade, distribuição gratuita de óculos e satisfação dos gestores e usuários, certamente faz dessa região, um exemplo de atendimento oftalmológico.

No campo administrativo, a Clínica Oftalmológica vem buscando cada vez mais estruturar seu banco de dados sobre vários aspectos, como número e tipo de atendimentos, diagnósticos, tratamento e procedimentos realizados. É também meta da administração elaborar banco de dados digital, com publicações científicas do próprio serviço.

O Hospital de Divinolândia tem recebido vários prêmios do Ministério da Saúde, pelos serviços prestados. O primeiro foi em 2001, no setor de Qualidade Hospitalar, dentre 1.421 hospitais nacionais avaliados, o Conderg ocupou o $7^{\circ}$ lugar. Em 2010 recebeu o título da Secretaria da Saúde do Estado de São Paulo na Qualidade Hospitalar; dentre 630 hospitais avaliados, o Conderg ocupou novamente o $7^{\circ} \operatorname{lugar}^{(23)}$.

A avaliação muito satisfatória quanto às expectativas de gestores municipais e usuários mostra a viabilidade de se manterem e criarem novos centros de atendimento financiados pelo SUS e associados a Hospitais Escola, com a finalidade de atender à demanda reprimida da região, manter excelente qualidade de serviço, servir de complementação da residência médica.

\section{CONCLUSÃO}

Na percepção dos gestores e usuários da clínica oftalmológica, gerida pelo consórcio de Municípios (Conderg) e serviço de Oftalmologia da Unicamp, o atendimento tem correspondido amplamente as expectativas.

\section{ABSTRACT}

Purpose: This study aimed to evaluate the assistance quality through the perception of the users and municipal health managers (mayors, health secretaries and screening team). Methods: A transversal and descriptive study was carried out. Results: The sample was comprised by 359 users and 48 managers. Medical assistance was considered excellent by $79.6 \%$ of the users, $93.7 \%$ of the managers, $87.5 \%$ of the health secretaries and $100 \%$ of the screening team. Reception received a great evaluation by $73.8 \%$ of the users and $93.8 \%$ of the selectors. Conclusion: The assistance model used at the Ophthalmologic Clinic of Divinolândia obtained a high level of satisfaction pleasing both users and managers.

Keywords: Health facilities; Health administration; Community health services; Public assistance; Control of quality; Consumer satisfaction

\section{REFERÊNCIAS}

1. Vuori H. Research needs in quality assurance. Qual Assur Health Care. 1989; 1(2-3):147-59.

2. Arkeman M. Avaliação dos serviços de saúde: avaliar o quê? Cad Saúde Pública. 1992;8(4):361-5.

3. Esperidião M. Avaliação da satisfação de usuários. Ciênc Saúde Coletiva. 2005; 10(Supl.):303-12

4. Convênio celebrado entre Unicamp e Consórcio de Desenvolvimento da Região de Governo de São João da Boa Vista - Conderg. Estatuto do CONDERG. Processo nº 9311/87. Divinolândia: CONDERG; 1988. p.2.

5. Kara-José N, Arieta CEL. Criação de serviço oftalmológico de referência. In KaraJosé N, Delgado AM, Arieta CEL, Rodrigues MLV, Alves MR. Prevenção da cegueira por catarata: Campinas: São Paulo; Ed da Unicamp; 1996. p.71-6.

6. Resnikoff S, Pascolini D, Mariotti SP, Pokharel GP. Global magnitude of visual impairment caused by uncorrected refractive errors in 2004. Bull World Health Organ. 2008;86(1):63-70.

7. Vitale S, Cotch MF, Sperduto RD. Prevalence of visual impairment in the United States. JAMA. 2006;295(18):2158-63.

8. Kara-José N, De Senne FMB, Arieta CEL, Delgado AM. Banco de óculos e laboratório óptico. Arq Bras Oftalmol.1996;59(3):316-8.

9. Jatene A. Novo modelo de saúde. Estudos Avançados.1999;13(35):51-64.

10. Machado MC, Kara-José N. Acesso à correção óptica: Experiência de óptica em Hospital Público de Divinolândia - São Paulo. In: Kara-José N, Rodrigues MLV. Saúde ocular e prevenção da cegueira. Rio de Janeiro:Cultura Médica; 2009. p.304-5.

11. Machado MC, Lourenço JLG, Figueiredo W, Kara-José N. Óculos de baixo custo: experiência em Divinolândia (SP). Arq Bras Oftalmol. 2010;73(1):57-9.

12. Tanaka OU, Melo C. Avaliação de Programas de Saúde do Adolescente: um modo de fazer. São Paulo: EDUSP; 2001.

13. O Consórcio de Desenvolvimento da Região de Governo de São João da Boa Vista - Uma Forma de Resolver a Saúde Conjuntamente. In: Fundação Prefeito Faria Lima Cepam. Coordenadoria de Gestão de Politicas Públicas - Cogepp. Municípios paulistas em busca de novas práticas: as 12 finalistas do prêmio Chopin Tavares de Lima - novas práticas municipais. São Paulo; 2005. p.99-110.

14. Sitzia J, Wood N. Patient satisfaction: A review of issues and concepts. Soc Sci Med. 1997;45(12):1829-43. 
15. Ware JE, Snyder MK, Wright WR, Davies AR. Defining and measuring patient satisfaction with medical care. Eval Program Plann. 1983;6(3-4):247-63.

16. Lëchner P, Perrealut M. Developpmente et validation d'une echellemulti-dimensiondlede satisfaction de patients de services d'hospitalisation en psychiatrie Rapport final. Société Canadienne d'Évaluation, Montreal. 1990.

17. Gomes MES, Barbosa EF. A técnica de grupos focais para obtenção de dados qualitativos. Educativa. 1999 (fev).

18. Owens DJ, Bachelor C. Patient satisfaction and the elderly. Soc Sci Med. 1996; 42(11):1483-91.
19. Bernhart MH, Wiadnyana IG, Whardjo H, Pohan I. Patient satisfaction in developing countries. Soc Sci Med. 1999;48(8):989-96.

20. Elias PE, Ferreira CW, Alves MCG, Cohn A, Kishima V, Escrivão Junior A et al. Atenção Básica em Saúde: comparação entre PSF e UBS por estrato de exclusão social no município de São Paulo. Ciênc Saúde Coletiva. 2006;11(3):633-41.

21. Gullo RM, Bernal VP, Kara-Jose N. Condições visuais de pacientes glaucomatosos em um hospital universitário. Arq Bras Oftalmol. 1996;59(2):147-50.

22. Secretaria Estadual de Saúde de São Paulo [Internet]. [citado 2010 Maio 12] Disponível em: www.saude.sp.gov.br

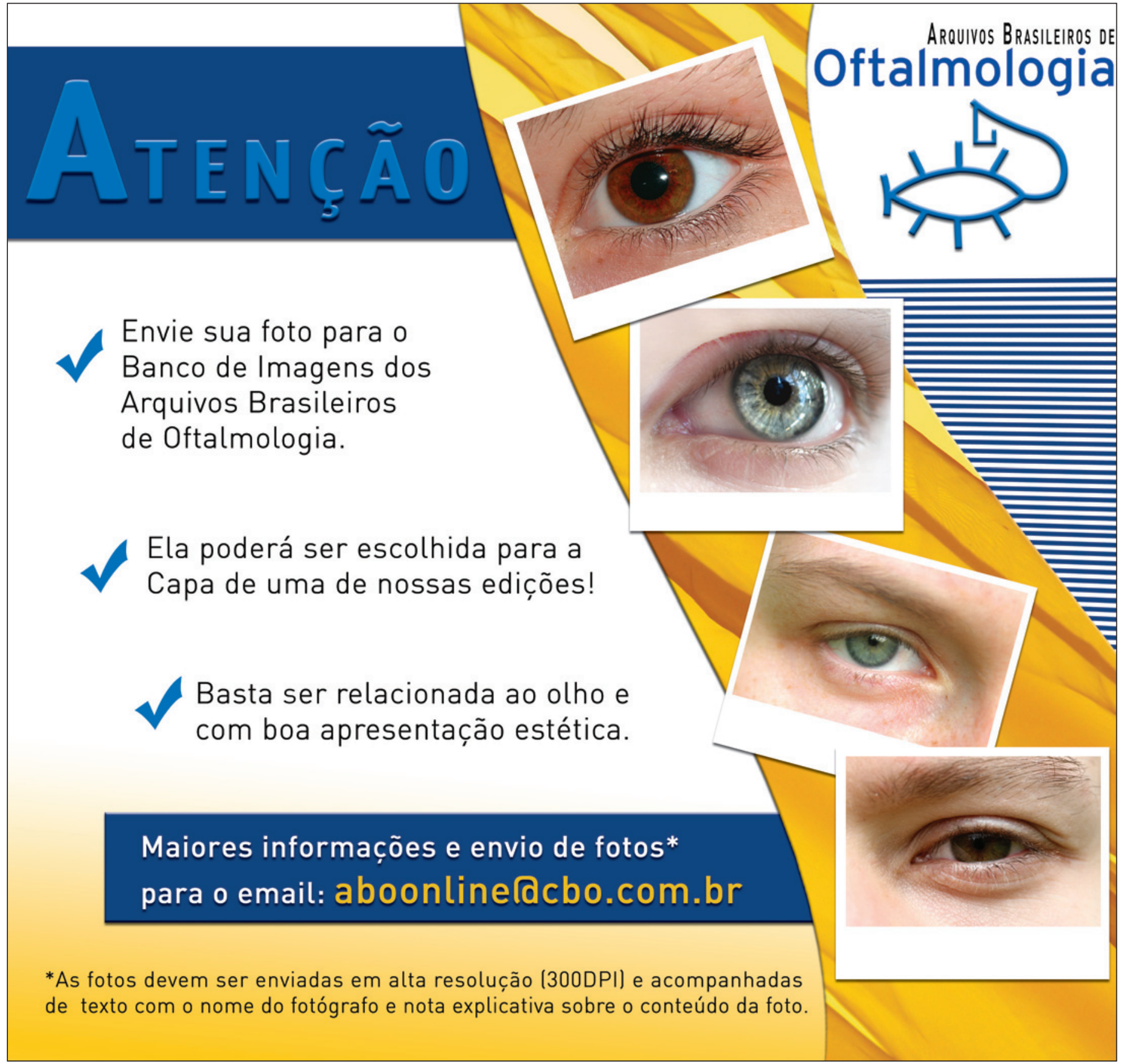

